\title{
Has-miR-125a and 125b are induced by treatment with cisplatin in nasopharyngeal carcinoma and inhibit apoptosis in a p53-dependent manner by targeting p53 mRNA
}

\author{
JIAN JUN CHEN ${ }^{1,2}$, SHI XI LIU ${ }^{3}$, MIAN ZHI CHEN ${ }^{3}$ and ZI YI ZHAO ${ }^{2}$ \\ ${ }^{1}$ Department of Medical, The First People's Hospital of Neijiang, Neijiang, Sichuan 641000; \\ ${ }^{2}$ Central Laboratory, Teaching Hospital of Chengdu University of Traditional Chinese Medicine, Chengdu, Sichuan 610000; \\ ${ }^{3}$ Department of E.N.T, West China Hospital, Sichuan University, Chengdu, Sichuan 610041, P.R. China
}

Received August 8,2014; Accepted April 22, 2015

DOI: $10.3892 / \mathrm{mmr} .2015 .3863$

\begin{abstract}
MicroRNA (miRNA) is a class of non-coding RNA, which targets mRNAs of interest and suppresses its expression by degradation or translational inhibition. miRNA (miR)-125a and miR-125b were previously demonstrated to translationally and transcriptionally inhibit the expression of p53. The observed downregulation of the protein level of p53 in cisplatin-treated patients with nasopharyngeal carcinoma (NPC) indicates the association between cisplatin resistance, miR-125a and miR-125b. In the present study, through the detection of the expression levels of miR-125a and miR-125b, a significant upregulation of these miRs was demonstrated in cisplatin-treated patients with NPC. As a consequence, the protein expression level of p53 decreased notably. To confirm the induction of miR-125a and miR-125b by treatment with cisplatin, a cisplatin-resistant TW03 cell model (TW03/DDP) was constructed. As expected, in the TW03/DDP cells, the expression levels of miR-125a and miR-125b were upregulated, and this caused downregulation of p53. Ectopic expression of these miRNAs in the TW03 cell model sensitized TW03 to cisplatin by decreasing the protein expression levels of p53, whereas ectopic expression in the antisense oligos of these microRNAs demonstrated the opposite effect. In addition, the present demonstrated that the cisplatin-induced expression of miR-125a and miR-125b inhibited cisplatin-induced apoptosis in the TW03 cells by decreasing the protein expression levels of p53. Taken together, the present study revealed for the first time, to the best of our knowledge, that induction of the expression of miR-125a and miR-125b by treatment with cisplatin resulted in resistance to the cisplatin drug in the NPC cells.
\end{abstract}

Correspondence to: Dr Zi Yi Zhao, Central Laboratory, Teaching Hospital of Chengdu University of Traditional Chinese Medicine, 39 Shierqiao Road, Jinniu, Chengdu, Sichuan 610000, P.R. China E-mail: zhaoziyi925@163.com

Key words: has-miR-125a, has-miR-125b, p53, nasopharyngeal carcinoma, TW03

\section{Introduction}

Nasopharyngeal carcinoma (NPC), which occurs predominantly in southeast China and southeast Asia, is considered a result of infection with Epstein-Barr virus, caused by the protein expression of LMP1 in this virus. Although radiotherapy and chemotherapy are widely used for the treatment of NPC and achieve a cure rate of $\sim 70 \%(1,2)$, the development of distant metastases develop remains a problem in $\sim 30 \%$ of patients, who have a poor prognosis (3). Cisplatin is widely used for NPC chemotherapy due to its effect of apoptosis induction in a p53-dependent and p53-independent manner (4). However, following six cycles of cisplatin-based chemotherapy, drug resistance against cisplatin develops in the majority of cases (5). The molecular mechanism underlying the resistance to cisplatin remains to be fully elucidated. The protein expression level of p53 is reported to be downregulated in human ovarian cancer cells resistant to cisplatin $(6,7)$, indicating an association between the protein expression level of p53 and cisplatin-resistance.

Cisplatin is one of the most widely used chemotherapeutic drugs for different types of cancer and solid tumor, including cancer of the ovary, neck, lung, head and testis (8). The anticancer effect of cisplatin predominantly occurs in two ways, via apoptotic pathway or cell cycle arrest in a p53-dependent manner or via p53-independent apoptosis (9-12). The way in which the damage induced by cisplatin on the genomic DNA triggers apoptosis remains to be elucidated. The tumor suppressor p53 has been implicated in this mechanism (13), however, the downstream effectors of cisplatin-induced p53-mediated apoptosis remain to be fully elucidated.

MicroRNA (miRNA) are a class of $\sim 22$ nucleotide, small, non-coding RNAs, which act as important post-transcriptional regulators of gene expression through translational repression or transcript cleavage (14). miRNAs regulate a number of basic cellular processes, and the clinical relevance of these malignancy-associated expression patterns, for example miRNA (miR)-125, has been reported. In humans, miR-125 is located on chromosomes 19,11 and 21 , and is expressed as two isoforms: miR-125a and miR-125b. Previous reports have demonstrated that miR-125a inhibits the proliferation of human 
gastric cancer cells in combination with trastuzumab (15). It is also associated with the migration and invasion of non-small cell lung cancer (16). miR-125 functions through the post-translational regulation of its several confirmed target genes, including ERBB2/3 and BAK1. Of all its targets genes, p53 is the gene of interest in the present study (17).

The present study assessed the induction of miR-125a and miR-125b following chemotherapy with cisplatin, which indicated that the overexpression of these two microRNAs contributed to chemoresistance in NPC by modifying the p53 pathway. Overall, the results suggested a novel mode of drug resistance to cisplatin, in a p53-dependent manner, to overcome cisplatin-induced apoptosis.

\section{Materials and methods}

Cell culture and treatment. The TW03, CNE-1 and CNE-2 NPC cell lines were obtained from American Type Culture Collection (ATCC; Manassas, VA, USA). NP69 (ATCC), a normal nasopharyngeal epithelial cell line, which was transformed with SV40 large T antigen, was frozen in liquid nitrogen. A TW03-derived cisplatin (DDP)-resistant subline was induced by exposure of the cells to Dulbecco's modified Eagle's medium (Gibco Life Technologies, Carlsbad, CA, USA), containing DDP. The TW03 cells were exposed to $300 \mathrm{nM}$ cisplatin and incubated for 3 weeks. A few colonies appeared and were trypsinized for further incubation in the presence of cisplatin. Over a period of 9 months, the concentration of cisplatin was increased to $20 \mu \mathrm{M}$ and the resultant cells were maintained in $10 \mu \mathrm{M}$ cisplatin. The CNE-1, CNE-2 and NP69 cells were cultured in Dulbecco's modified Eagle's medium, containing $10 \%$ fetal bovine serum, in a humidified atmosphere with $5 \% \mathrm{CO}_{2}$ at $37^{\circ} \mathrm{C}$.

The present study was approved by the ethics committee of the local institutional review board at the First People's Hospital of Neijiang (Sichuan, China). Written informed consent was obtained from all the patients prior to all clinical investigations.

Human samples. Fresh cancerous nasopharyngeal tissues and matched adjacent nasopharyngeal tissues were obtained from 10 patients, who had received primary NPC resection during one year, at West China Hospital (Sichuan, China). A total of 10 patients with NPC (4 males and 6 females, mean age, $54.6 \pm 6.8$ years) were recruited. All patients exhibited stage III and IV, without distant metastasis. All patients were randomly selected and had been treated with six cycles of platinum-based chemotherapy. One sample with a diameter between 2 and $4 \mathrm{~cm}$ was obtained from each patient. The samples were placed immediately in liquid nitrogen following surgical removal and were stored at $-80^{\circ} \mathrm{C}$ prior to use.

Transfection. The TW03 cells were seeded into 12-well plates at a density of $5 \times 10^{5}$ for $24 \mathrm{~h}$ prior to transfection, and were subsequently transfected with Lipofectamine 2000 (Invitrogen Life Technologies, Carlsbad, CA, USA). Following incubation for $48 \mathrm{~h}$, the transfected TW03 or TW03/DDP were washed with ice-cold phosphate-buffered saline and were harvested for further analysis. mirVana ${ }^{\circledR}$ miRNA mimic for miR-125a (cat. no. MC12378), miR-125b (cat. no. MC10148) and mirVana ${ }^{\circledR}$ miRNA inhibitor for miR-125a (cat. no. 12378) and miR-125b (cat. no. 10148) were purchased from Life Technologies (Carlsbad, CA, USA), and the concentration used was $5 \mathrm{nM}$, unless stated otherwise.

$R N A$ extraction and reverse transcription-quantitative polymerase chain reaction $(R T-q P C R)$ analysis of miRNAs. The total RNA was extracted from the patient samples or cultured cells using TRIzol (Life Technologies), and the quantity and quality of the extracted total RNA was analyzed by measuring the absorbance at $260 \mathrm{~nm}$. A miScript SYBR ${ }^{\circledR}$ Green PCR kit from Qiagen (Mississauga, ON, Canada) was used for RT-qPCR analysis. The primers for miR-125a, miR-125b and U6 snRNA (internal control) were purchased from Qiagen. The fold-changes in miRNA and mRNA in the NPC tissues relative to the adjacent non-tumor tissues were calculated using the $2^{-\triangle \Delta C T}$ method (18). To assess the relative mRNA fold changes, GAPDH was used as the internal control for mRNA expression. Each PCR was performed in triplicate.

Measurement of cell viability using a cholecytokinin octapeptide (CCK8) assay. The transfected TW03 cells were seeded into 96 -well plates at a density of $2.5 \times 10^{4}$ cells/well. Following treatment or transfection of the cells, DDP $(1-15 \mu \mathrm{M})$ was added to the medium for long-term incubation at 24, 48 or $72 \mathrm{~h}$, and CCK8 reagent (Sigma-Aldrich, St. Louis, MO, USA; $10 \mu \mathrm{l}$ ) was added to each well for $4 \mathrm{~h}$ at $37^{\circ} \mathrm{C}$. The absorbance was subsequently measured between 450 and $590 \mathrm{~nm}$ using a multiscan FC (Thermo Fisher Scientific, Waltham, MA, USA).

Western blotting. The total protein of the cellular lysate was extracted using ProteoPrep ${ }^{\circledR}$ Total Extraction Sample kit (Sigma-Aldrich) and was quantitated using a Bicinchoninic Acid kit. Following separation of $10 \mu \mathrm{g}$ protein by electrophoresis on 4-12\% SDS-PAGE gel, the proteins were transferred onto polyvinylidene fluoride membranes (Bio-Rad Laboratories, Inc., Hercules, CA, USA). The membranes were subsequently blocked with PBS, containing 5\% BSA and following washing in PBS three times, the membranes were exposed to primary antibody against p53 Do-1 (mouse monoclonal; 1:1000; cat. no. ab1101; Abcam, Cambridge, England) and rabbit anti-mouse IgG secondary antibody (1:5,000, cat. no. ab6728; Abcam). The signals were visualized by chemiluminescence using enhanced chemilluminescence Dura Extended Duration Substrate (Pierce, Rockford, IL, USA).

Soft agar assay. Soft agar assays were performed to determine the in vitro tumorigenicity of the cells. The cells $\left(\sim 5 \times 10^{2}\right)$ were seeded into $0.3 \%$ agarose, with a $0.6 \%$ agarose underlay, in a six-well plate. The plates were maintained at $37^{\circ} \mathrm{C}$ in an atmosphere of $5 \% \mathrm{CO}_{2}$ for 3 weeks. The number of cell colonies were counted by two independent observers. The experiments were performed in triplicate, with the data presented as the mean of three repeated experiments.

Statistical analyses. The data from at least three independent experiments are expressed as the mean \pm standard deviation. An analysis of variance or t-test was used, when appropriate, to examine the differences in expressions under the different 
A

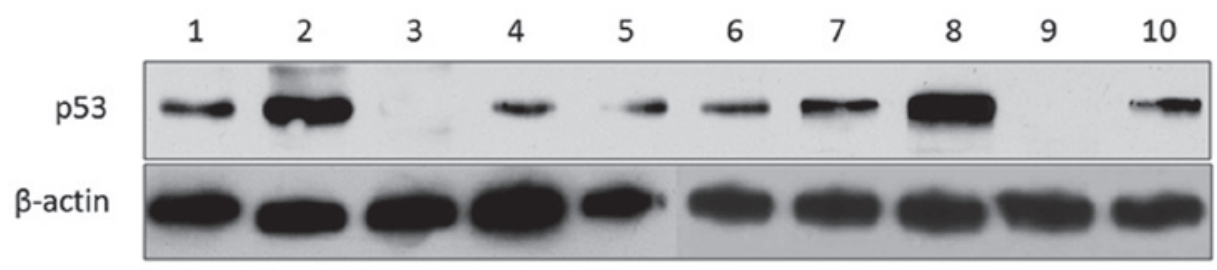

B

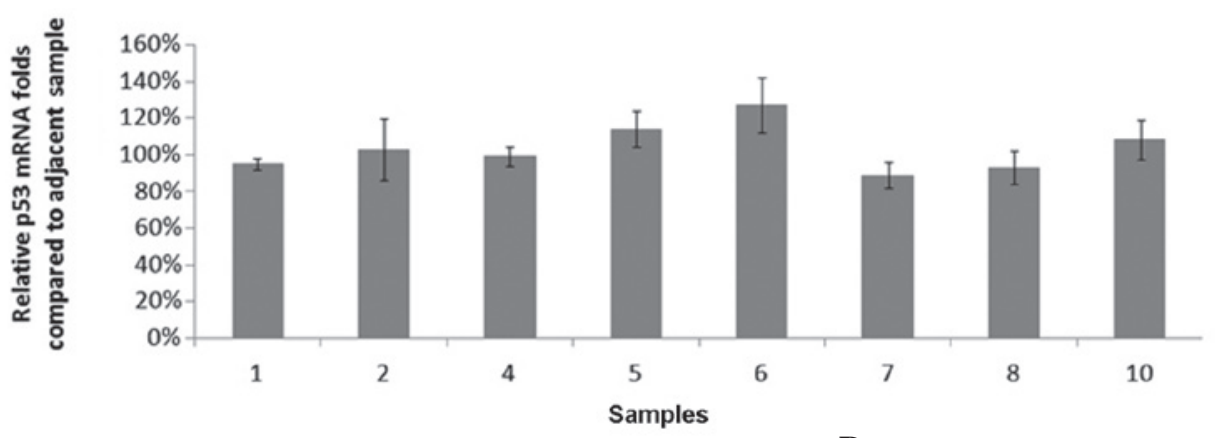

C

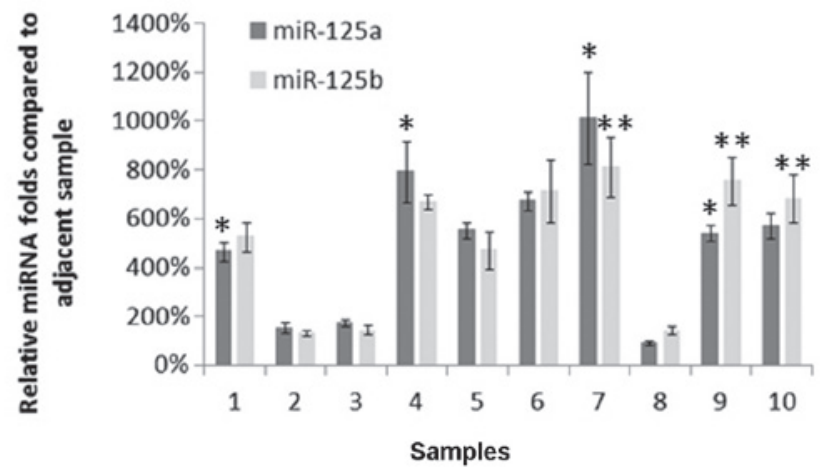

D

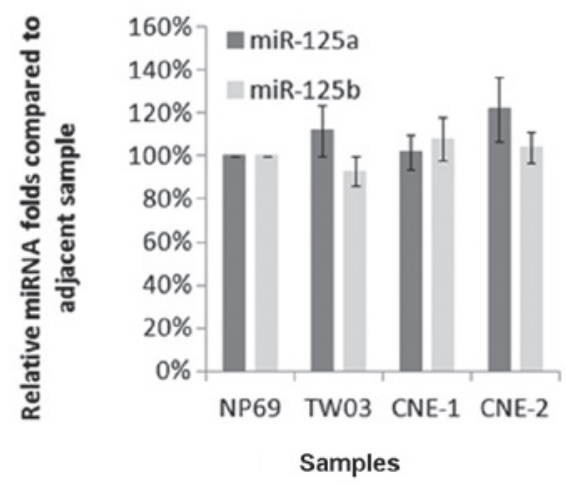

Figure 1. Relative protein expression levels of p53, miR-125a and miR-125b in NPC tissues and different NSCLC cell lines using semi-quantitative western blotting and RT-qPCR. (A) p53 protein and (B) mRNA profile in NPC tissues, compared with normal adjacent tissues. (C) Expression levels of miR-125a and miR-125b in NSCLC tissues, compared with normal adjacent tissues. (D) Relative expression levels of miR-125a and miR-125b in different NPC cell lines, determined using RT-qPCR. The relative expression levels were calculated using the $2^{-\Delta \Delta C t}$ method. Data are expressed as the mean \pm standard deviation $\left({ }^{*} \mathrm{P}<0.05\right.$ and ${ }^{* *} \mathrm{P}<0.01$, vs. adjacent sample). NPC, nasopharyngeal carcinoma; NSCLC, non-small cell lung carcinoma; RT-qPCR, reverse transcription-quantitative polymerase chain reaction.

conditions. $\mathrm{P}<0.05$ was considered to indicate a statistically significant difference. The statistical software package SAS 9.2 (SAS Institute, Inc., Cary, NC, USA) was used for all statistical analyses.

\section{Results}

Has-miR-125a and $125 b$ are significantly co-upregulated in human NPC tissues. The previous finding that the protein expression of p53 significantly decreased in cisplatin-treated patients with ovarian cancer prompted the present study to determine the protein expression levels of p53 in patients with NPC (19). The NPC samples and matched normal tissues were collected using laser capture micro-dissection and the expression levels of p53 in the two were determined using RT-qPCR and semi-quantitative western blotting. The results demonstrated that the protein expression level of p53 was downregulated in $80 \%(8 / 10)$ and mutated in $20 \%(2 / 10)$ of the patients with NPC, without altering the levels of mRNA expression (Fig. 1A and B).

The downregulation of the protein expression levels of p53 may have been due to the regulation of miRNA. To examine this possibility, the same pairs of samples were analyzed for the expression of miR-125a and miR-125b, which target the mRNA of p53. As shown in Fig. 1C, miR-125a and miR-125b were upregulated in 7/10 of the NPC samples, compared with the adjacent samples. The expression levels of miR-125a and miR-125b did not differ significantly among age, gender, tumor-node-metastasis staging or metastasis in the 10 NPC samples (data not shown). To assess the biological role of miR-125a and miR-125b in a panel of NPC cell lines, the expression of these two miRNAs were detected in the TW03, CNE-1 and CNE-2 lines using RT-qPCR. However, compared with the NP69 human nasal epithelial cell, neither miR-125a nor miR-125b exhibited significant changes, indicating that the overexpression of miR-125a and miR-125b were induced (Fig. 1D).

Confirmation of cisplatin-induced upregulation of $\mathrm{miR}-125 \mathrm{a}$ and miR-125b in cisplatin-resistant NPC TW03 cells, using $R T-q P C R$ and expression levels of $p 53$. The TW03 human NPC cell line was used for the selection of cisplatin resistance for the endogenous wild-type p53 and low level of intrinsic resistance to cisplatin. The inhibition of cell viability, shown in Fig. 2A, 
A

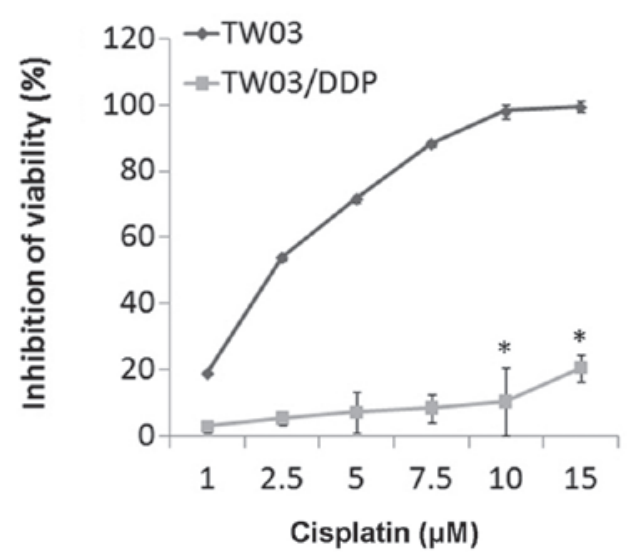

C

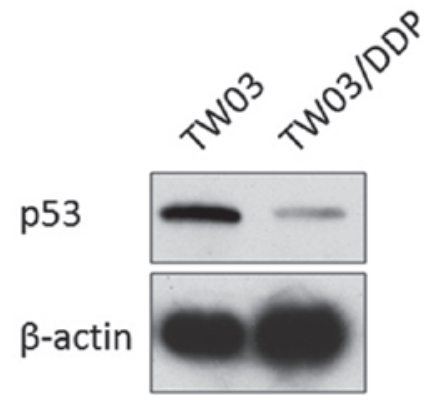

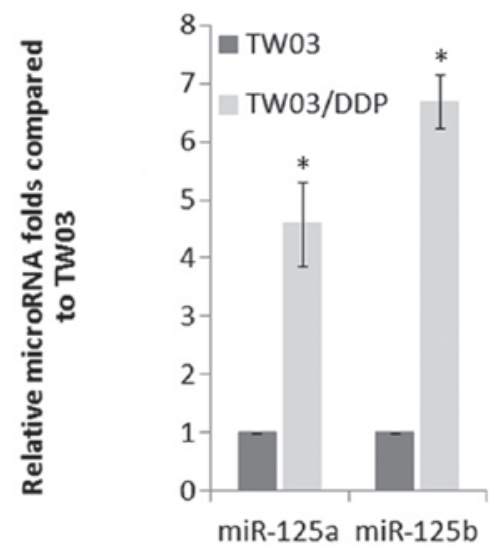

D

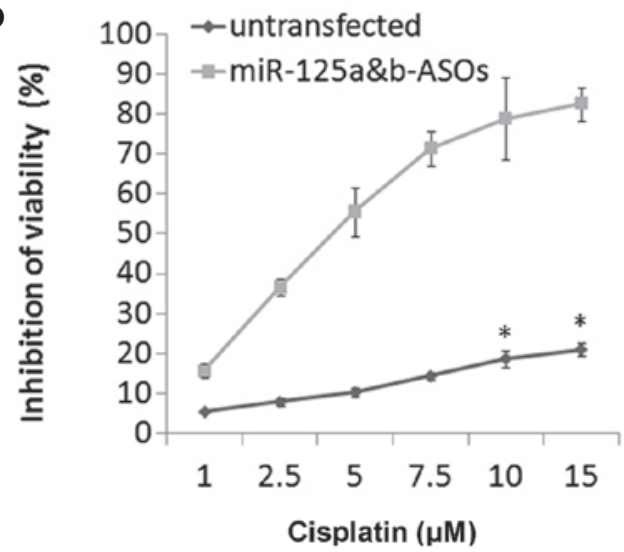

Figure 2. miR-125a- and miR-125b-induced cisplatin resistance in TW03 cells by decreasing the protein expression of p53. (A) Confirmation of cisplatin resistance in the TW03/DDP cells. (B) Expression levels of miR-125a and miR-125b in the TW03/DDP cells were detected using reverse transcription-quantitative polymerase chain reaction. (C) Downregulation of p53 in cisplatin-resistant TW03 was detected using western blotting. (D) Elimination of cisplatin resistance via overexpressing miR-125a and miR-125b ASOs. ("P<0.05, vs. untreated TWO3 cells or untransfected cells). miR, microRNA; ASO, DDP, cisplatin.

indicated the relative resistance level of the TW03/DDP cells for cisplatin, which was higher compared with the parental TW03 cell line. Further detection of miR-125a and miR-125b and their target gene p53 (Fig. 2B and C) indicated significant induction of miR-125a and miR-125b, and downregulation of the protein expression level of p53, without affecting the mRNA expression of p53 (data not shown).

To further examine the effects of miR-125a and miR-125b on the expression levels of p53 and sensitivity to cisplatin, TW03/DDP cells were transfected with miR-125a and miR-125b antisense oligos (ASOs). The protein expression levels of p53 and inhibition of cell viability in these cells were determined and the results demonstrated that miR-125a and miR-125b induced the downregulation of p53 post-transcriptionally, and sensitized TW03/DDP to cisplatin (Fig. 2D), suggesting that the presence of miR-125a and miR-125b prompted cisplatin sensitivity.

Cisplatin-induced miR-125a and miR-125b post-translationally inhibit the expression of p53 and cisplatin-induced apoptosis, and promote proliferation and colony formation. The present study subsequently investigated whether inhibiting or increasing the expression levels of miR-125a and miR-125b modulated cell survival or the sensitivity of TW03 cells to cisplatin, which is currently used for chemotherapy.
Following stable transfection of the mixture of miR-125a and miR-125b mimics into the TW03 cells, the cells were treated with a series of concentrations of cisplatin. The effect of miR-125a and miR-125b on the drug resistance of TW03 cells is shown in Fig. 3A. The results indicated that the overexpression of miR-125a and miR-125b notably reduced the drug sensitivity of TW03 cells to cisplatin. The growth inhibition of the TW03 cells by cisplatin was significantly increased by transfection with miR-125a and miR-125b ASOs (Fig. 3B).

Considering the multiple functions of $\mathrm{p} 53$, the present study further assessed the proliferation rate and colony formation regulated by p53 in TW03/DDP cells overexpressing miR-125a and miR-125b. In the absence of cisplatin, downregulation of p53 induced colony formation on soft agar, suggesting that the effects of miR-125 on p53 are not only in cisplatin sensitization (Fig. 3C and D).

\section{Discussion}

It has been previously demonstrated that the expression of miRNAs is significantly regulated in NPC and is widely involved in malignant progression $(20,21)$. Although abnormal miRNA expression has been reported in several types of cancer, promoting investigation to understand the 
A

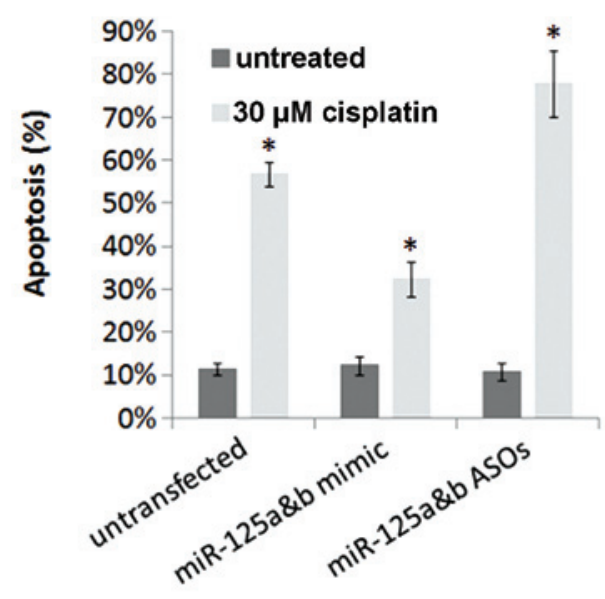

C

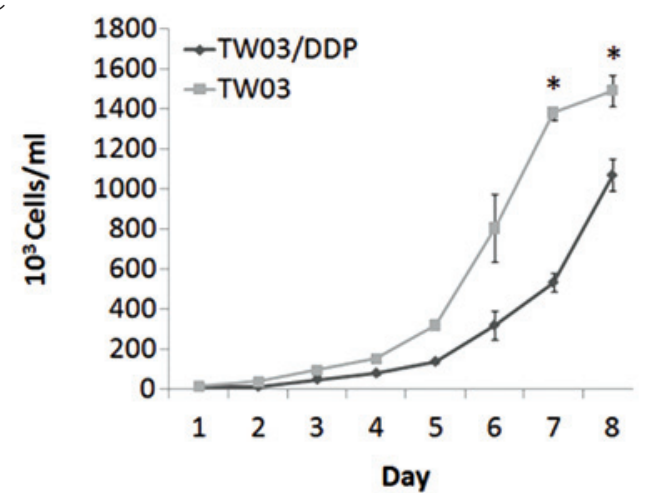

B

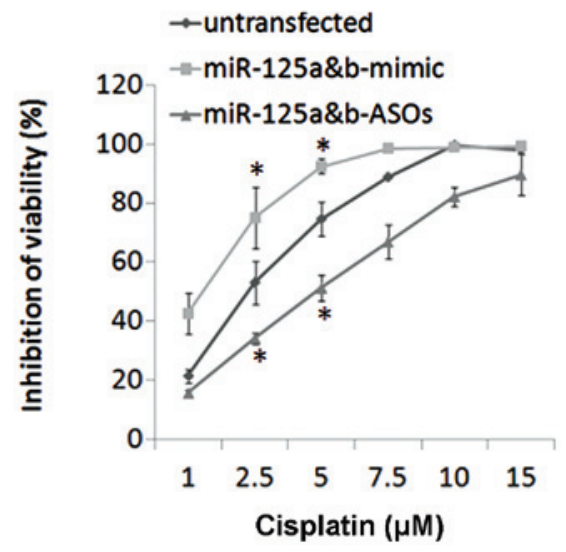

D

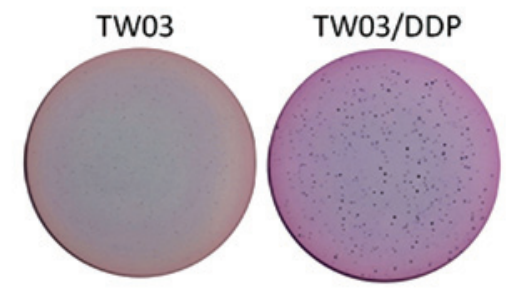

Figure 3. Overexpression of miR-125a and miR-125b indues cisplatin resistance, inhibits cisplatin-induced apoptosis and promotes cell proliferation and colony formation. (A and B) miR-125a and miR-125b induced cisplatin resistance by inhibiting cell apoptosis. (C) Proliferation and (D) colony formation were promoted in the cisplatin-resistant TW03 cells. Colonies were stained with $100 \mathrm{mg} / \mathrm{ml} \mathrm{NBT}$ at $37^{\circ} \mathrm{C}$ for $4 \mathrm{~h}$ and were scanned using an $\mathrm{HP}$ Scanjet 4850 photo scanner and the colonies were counted. ( ${ }^{*} \mathrm{P}<0.05$, vs. untreated TW03 cells). miR, microRNA; DDP, cisplatin.

roles of miRNAs in modulating cancer development, the hundreds of potential targets involved in several signal transduction pathways mean the functions of microRNAs in the carcinogenesis of NPC remain to be fully elucidated (22). In addition to the well-understood roles of miRNAs, previous studies have demonstrated the association of microRNAs to drug resistance (23). Evidence has revealed the association between miRNA and drug resistance as a novel strategy for therapeutic intervention by targeting miRNAs (24).

Previous studies have demonstrated that the expression levels of miR-125a and miR-125b are downregulated in several types of cancer, indicating the tight association of these miRNAs with cancer (25). The results of human miRNA microarrays have indicated that NPC tissues exhibit lower expression levels of miR-125a and miR-125b. These previous studies rarely focused on the drug resistance and expression levels of miR-125a and miR125b in NPC samples from patients. The present studydemonstrated for the first time, to the best of our knowledge, that miR-125a and miR-125b were frequently upregulated in cisplatin-resistant NPC tissues, compared with the matched adjacent normal tissues. In addition, the upregulation of miR-125a and miR-125b were significantly associated with downregulation in the protein expression of p53. Notably, the expression levels of miR-125a and miR-125b in the NPC cell lines were almost identical, compared with the NP69 human nasal epithelial cell line, which led $t$ to further assessment of the association betweem cisplatin resistance and the upregulation of miR-125a and miR-125b. Consistent with the results observed in the NPC samples, the established cisplatin-resistant NPC cell line also demonstrated significantly lower expression levels of miR-125a and miR-125b. Ectopic expression of miR-125a and miR-125b downregulated the protein expression of $\mathrm{p} 53$. By contrast, expression of miR-125a and miR-125b ASOs upregulated the protein expression of p53 and sensitized the TW03/DDP cells to cisplatin. Consistently, the ectopic expression of miR-125a and miR-125b decreased the apoptotic rate following treatment with cisplatin. However, the underlying mechanism of miR-125a and miR-125b remains to be elucidated.

In conclusion, the present study indicated two important findings. Firstly, drug resistance in the NPC samples, induced by cisplatin treatment, upregulated the expression levels of miR-125a and miR-125b. Secondly, the upregulation of miR-125a and miR-125b caused drug resistance by targeting p53 mRNA and subsequently decreasing the protein expression level. This indicated a possible mechanism of drug resistance caused by miR-125a and miR-125b in a p53-dependent manner. These findings may be useful for the development of novel targets to avoid drug resistance. 


\section{Acknowledgements}

The authors would like to thank Dr Chang-jin Chen from Chengdu University of Traditional Chinese Medicine (Chengdu, China) for suggestions throughout the present study. This study was supported by a Sichuan provincial scientific grant (no. 2012SZZ011).

\section{References}

1. Wei WI and Sham JS: Nasopharyngeal carcinoma. Lancet 365: 2041-2054, 2005.

2. Cao SM, Simons MJ and Qian CN: The prevalence and prevention of nasopharyngeal carcinoma in China. Chin J Cancer 30: 114-119, 2011.

3. Langendijk JA, Leemans CR, Buter J, Berkhof J and Slotman BJ: The additional value of chemotherapy to radiotherapy in locally advanced nasopharyngeal carcinoma: a meta-analysis of the published literature. J Clin Oncol 22: 4604-4012, 2004.

4. Fajac A, Da Silva J, Ahomadegbe JC, Rateau JG, Bernaudin JF, Riou G and Bénard J: Cisplatin-induced apoptosis and p53 gene status in a cisplatin-resistant human ovarian carcinoma cell line. Int J Cancer 68: 67-74, 1996.

5. Jin Y, Cai XY, Shi YX, Xia XY, Cai YC, Cao Y, Zhang WD, $\mathrm{Hu}$ WH and Jiang WQ: Comparison of five cisplatin-based regimens frequently used as the first-line protocols in metastatic nasopharyngeal carcinoma. J Cancer Res Clin Oncol 138: $1717-1725,2012$

6. Fraser M, Bai T and Tsang BK: Akt promotes cisplatin resistance in human ovarian cancer cells through inhibition of p53 phosphorylation and nuclear function. Int J Cancer 122: 534-546, 2008.

7. Leung EL, Fraser M, Fiscus RR and Tsang BK: Cisplatin alters nitric oxide synthase levels in human ovarian cancer cells: involvement in p53 regulation and cisplatin resistance. $\mathrm{Br} \mathbf{J}$ Cancer 98: 1803-1809, 2008.

8. Galluzzi L, Vitale I, Michels J, Brenner C, Szabadkai G, Harel-Bellan A, Castedo M and Kroemer G: Systems biology of cisplatin resistance: Past, present and future. Cell Death Dis 5: $1257-1274,2014$.

9. Zamble DB, Jacks T and Lippard SJ: p53-Dependent and-independent responses to cisplatin in mouse testicular teratocarcinoma cells. Proc Natl Acad Sci USA 95: 6163-6168, 1998

10. Bragado P, Armesilla A, Silva A and Porras A: Apoptosis by cisplatin requires p53 mediated p38alpha MAPK activation through ROS generation. Apoptosis 12: 1733-1742, 2007.

11. Moreno CS, Matyunina L, Dickerson EB, Schubert N, Bowen NJ, Logani S, Benigno BB and McDonald JF: Evidence that p53-mediated cell-cycle-arrest inhibits chemotherapeutic treatment of ovarian carcinomas. PLoS One 2: e441, 2007.
12. Petit T, Bearss DJ, Troyer DA Munoz RM and Windle JJ: p53-independent response to cisplatin and oxaliplatin in MMTV-ras mouse salivary tumors. Mol Cancer Ther 2: 165-171, 2003.

13. Michelle MR and Zahid HS: Resistance and gain-of-resistance phenotypes in cancers harboring wild-type p53. Biochem Pharmacol 83: 1049-1062, 2011.

14. Bartel DP: MicroRNAs: genomics, biogenesis, mechanism and function. Cell 116: 281-297, 2004.

15. Nishida N, Mimori K, Fabbri M, Yokobori T, Sudo T, Tanaka F, Shibata K, Ishii H, Doki Y and Mori M: MicroRNA-125a-5p is an independent prognostic factor in gastric cancer and inhibits the proliferation of human gastric cancer cells in combination with trastuzumab. Clin Cancer Res 17: 2725-2733, 2011.

16. Zhao YM, Zhou JM, Wang LR, He HW, Wang XL, Tao ZH, Sun HC, Wu WZ, Fan J, Tang ZY and Wang L: HIWI is associated with prognosis in patients with hepatocellular carcinoma after curative resection. Cancer 118: 2708-2717, 2012.

17. Le MT, Teh C, Shyh-Chang N, Xie H, Zhou B, Korzh V, Lodish HF and Lim B: MicroRNA-125b is a novel negative regulator of p53. Genes Dev 23: 862-876, 2009.

18. Rao X, Huang X, Zhou Z and Lin X: An improvement of the $2^{\wedge}$ (-delta delta CT) method for quantitative real-time polymerase chain reaction data analysis.Biostat Bioinforma Biomath 3: 71-85, 2013

19. Feudis PD, Debernardis D, Beccaglia P, Valenti M, Siré EG, Arzani D, Stanzione S, Parodi S, D'Incalci M, Russo P and Broggini M: DDP-induced cytotoxicity is not influenced by $\mathrm{p} 53$ in nine human ovarian cancer cell lines with different p53 status. Br J Cancer 76: 474-479, 1997.

20. Kovalchuk O, Filkowski J, Meservy J, Ilnytskyy Y, Tryndyak VP, Chekhun VF and Pogribny IP: Involvement of microRNA-451 in resistance of the MCF-7 breast cancer cells to chemotherapeutic drug doxorubicin. Mol Cancer Ther 7: 2152-2159, 2008.

21. Liang Z, Wu H, Xia J, Li Y, Zhang Y, Huang K, Wagar N, Yoon Y, Cho HT, Scala S and Shim H: Involvement of miR-326 in chemotherapy resistance of breast cancer through modulating expression of multidrugresistance-associated protein 1. Biochem Pharmacol 79: 817-824, 2010

22. Pogribny IP, Filkowski JN, Tryndyak VP, Golubov A, Shpyleva SI and Kovalchuk O: Alterations of microRNAs and their targets are associated with acquired resistance of MCF-7 breast cancer cells to cisplatin. Int J Cancer 127: 1785-1794, 2010.

23. Zhao JJ, Lin J, Yang H, Kong W, He L, Ma X, Coppola D and Cheng JQ: MicroRNA-221/222 negatively regulates estrogen receptor alpha and is associated with tamoxifen resistance in breast cancer. J Biol Chem 283: 31079-31086, 2008.

24. Sarkar FH, Li Y, Wang Z and Kong D: Novel targets for prostate cancer chemoprevention. Endocr Relat Cancer 17: 195-212, 2010.

25. Xu N, Brodin P, Wei T, Meisqen F, Eidsmo L, Nagy N, Kemeny L, Ståhle M, Sonkoly E and Pivarcsi A: mir-125b, a microRNA downregulated in psoriasis, modulates keratinocyte proliferation by targeting FGFR2. J Invest Dermatol 131: 1521-1529, 2011. 